\title{
BMJ Open Influence of chronic exercise on carotid atherosclerosis in marathon runners
}

\author{
Beth A Taylor, ${ }^{1,2}$ Amanda L Zaleski, ${ }^{1}$ Jeffrey A Capizzi, ${ }^{1}$ Kevin D Ballard, ${ }^{1}$ \\ Christopher Troyanos, ${ }^{3}$ Aaron L Baggish, ${ }^{4}$ Pierre A D'Hemecourt, ${ }^{3}$ Marcin R Dada, ${ }^{1}$ \\ Paul D Thompson ${ }^{1}$
}

To cite: Taylor BA, Zaleski AL, Capizzi JA, et al. Influence of chronic exercise on carotid atherosclerosis in marathon runners. BMJ Open 2014:4:e004498.

doi:10.1136/bmjopen-2013004498

- Prepublication history for this paper is available online. To view these files please visit the journal online (http://dx.doi.org/10.1136/ bmjopen-2013-004498).

Received 18 November 2013 Revised 10 January 2014 Accepted 17 January 2014

CrossMark

${ }^{1}$ Department of Cardiology, Henry Low Heart Center, Hartford Hospital, Hartford, Connecticut, USA

${ }^{2}$ Department of Health

Sciences, University of Hartford, Bloomfield, Connecticut, USA

${ }^{3}$ Children's Hospital, Boston, Massachusetts, USA

${ }^{4}$ Division of Cardiology, Massachusetts General

Hospital, Boston,

Massachusetts, USA

Correspondence to Dr Beth A Taylor; beth.taylor@hhchealth.org

\section{ABSTRACT}

Objectives: The effect of habitual, high-intensity exercise training on the progression of atherosclerosis is unclear. We assessed indices of vascular health (central systolic blood pressure (SBP) and arterial stiffness as well as carotid intima-medial thickness (cIMT)) in addition to cardiovascular risk factors of trained runners versus their untrained spouses or partners to evaluate the impact of exercise on the development of carotid atherosclerosis.

Setting: field study at Boston Marathon.

Participants: 42 qualifiers (mean age \pm SD: 46 \pm 13 years, 21 women) for the 2012 Boston Marathon and their sedentary domestic controls $(46 \pm 12$ years, $\mathrm{n}=21$ women).

Outcomes: We measured medical and running history, vital signs, anthropometrics, blood lipids, $C$ reactive protein (CRP), 10 years Framingham risk, central arterial stiffness and SBP and cIMT.

Results: Multiple cardiovascular risk factors, including CRP, non-high-density lipoprotein cholesterol, triglycerides, heart rate, body weight and body mass index (all $p<0.05$ ), were reduced in the runners. The left and right cIMT, as well as central SBP, were not different between the two groups (all $p>0.31$ ) and were associated with age (all $r \geq 0.41 ; p<0.01$ ) and Framingham risk score (all $r \geq 0.44 ; p<0.01$ ) independent of exercise group (all $p>0.08$ for interactions). The amplification of the central pressure waveform (augmentation pressure at heart rate $75 \mathrm{bpm}$ ) was also not different between the two groups $(p=0.07)$ but was related to age $(p<0.01)$ and group $(p=0.02)$ in a multiple linear regression model.

Conclusions: Habitual endurance exercise improves the cardiovascular risk profile, but does not reduce the magnitude of carotid atherosclerosis associated with age and cardiovascular risk factors.

\section{INTRODUCTION}

Carotid intima-medial thickness (cIMT) is a measurement of carotid atherosclerosis and predicts future vascular events such as stroke and heart attack. ${ }^{1}$ Moderate habitual physical activity is associated with reduced cardiovascular deaths, but it is not clear whether the

\section{Strengths and limitations of this study}

- Previous contrasting results on the impact of repetitive strenuous exercise on the development of atherosclerosis might be explained by the impact of multiple lifestyle factors on cardiovascular risk. For example, runners are likely to engage in other health behaviours (in addition to exercise) which could influence atherosclerotic processes and confound interpretation of data.

- Therefore, we have used a novel comparison of runners and their non-runner control spouses to conclude that habitual, high-intensity run training improves many aspects of the cardiovascular profile but does not reduce atherosclerosis measured by carotid intima-medial thickness (cIMT). Sustained high-intensity aerobic training does not reduce the magnitude of carotid atherosclerotic progression associated with age and disease, but does not appear to exacerbate it.

- We assessed atherosclerosis in our participants using cIMT, but other procedures such as coronary artery calcium score might provide a better assessment of coronary and cardiovascular disease risk. Our control participants were also not entirely sedentary. Controls performed less vigorous exercise, but they did perform similar amounts of moderate exercise as the runners. However, this design may enhance the validity of our study because it might better isolate the influence of habitual, high-intensity exercise training on cardiovascular risk and carotid atherosclerosis.

reduction in cardiac events is due to exercise-induced reductions in atherosclerotic risk factors and atherosclerosis or due to other factors such as enhanced vagal tone, increased electrical stability and a reduction in sudden death. ${ }^{2} 3$

Several studies have examined atherosclerotic burden in athletes. Galetta et $a l^{t}$ observed that cIMT was $46 \%$ thicker in older adults, but lower in older endurance-trained athletes than sedentary controls, and increased cardiorespiratory fitness is associated with reduced cIMT in healthy ${ }^{5}$ and 
diabetic $^{6} 7$ populations. In contrast, Heffernan et $a l^{8}$ found no significant differences in cIMT scores between exercise trained and age-matched, sedentary men with prehypertension. In addition, recent data showed that veteran marathon runners exhibit higher coronary artery calcium scores compared with non-running controls matched for Framingham risk scores ${ }^{9}$ and, similarly, male marathon runners display a surprisingly high prevalence of carotid and peripheral atherosclerosis. ${ }^{10} \mathrm{~A}$ recent editorial proposed that repeated bouts of sustained and/or high-intensity aerobic exercise, such as that required for marathon training and competition, evokes systemic vascular remodelling that shifts the effect of aerobic exercise from cardioprotective to atherogenic. ${ }^{11}$

These contrasting results on the impact of repetitive strenuous exercise on the development of atherosclerosis prompted a recent meta-analysis on the effects of exercise on carotid atherosclerosis to conclude that "it remains questionable whether long-term exercise can decelerate the development of carotid atherosclerosis." ${ }^{12}$ However, it is possible that discrepant results might also be explained by the impact of multiple lifestyle factors on cardiovascular risk. For example, runners are likely to engage in other health behaviours (in addition to exercise) which could influence atherosclerotic processes and confound interpretation of data.

Accordingly, the current study compared carotid atherosclerosis measured by cIMT and the cardiovascular risk of runners participating in the 2012 Boston Marathon versus non-running spouses/domestic partners living in the same household (to control for other lifestyle factors such as diet). In addition to cIMT, we also assessed central systolic blood pressure (SBP) and arterial stiffness (the amplification of the pressure waveform at the aorta), both of which contribute to central arterial stiffening, smooth muscle hypertrophy and increased intima-medial thickness. ${ }^{13}{ }^{14}$ We hypothesised that the runners would have a more favourable atherosclerotic risk profile and lower cIMT values than the non-runner controls.

\section{METHODS}

Forty-two runners (50\% women) registered for the 116th Boston Athletic Association Marathon (16 April 2012) and their non-running partners (married/committed and living in the same household) were recruited for the study. All runners had achieved the Boston Athletic Association's qualifying standard and were running the marathon except for two runners who were involved in training but not competing that year. Participants who smoked or diagnosed with cardiovascular or metabolic disease besides hypercholesterolaemia were excluded. Controls did not participate in regular, sweat-inducing physical activity $\geq 2$ times/week. Participants provided written, informed consent to participate.
The day before the race, the participants provided a medical and running history as well as their training mileage over the 3 months preceding the marathon. They also completed the Paffenbarger Physical Activity Questionnaire $^{15}$ to calculate average weekly hours of moderate and vigorous activity. Participants also completed the Block Food Screener ${ }^{16}$ to assess dietary intake. Resting blood pressure, heart rate (Welch Allen 52000 Vital Signs Monitor; Skaneateles Falls, New York, USA), height and body weight were measured. Venous blood was obtained after a $12 \mathrm{~h}$ fast to measure total and high-density lipoprotein cholesterol (HDL-C), triglycerides and $\mathrm{C}$ reactive protein (Quest Diagnostics Nichols Institute, Chantilly, Virginia, USA). Low-density lipoprotein cholesterol was estimated. ${ }^{17}$ Ten years Framingham risk was calculated according to the National Cholesterol Education Programme online calculator (http:// hp2010.nhlbihin.net/atpiii/calculator.asp).

cIMT was measured with Doppler ultrasound. The artery was imaged $1 \mathrm{~cm}$ distal to the right and left carotid bulb using a 5-12 MHz multifrequency lineararray transducer attached to a high-resolution ultrasound machine (Terason t3000; Burlington, Massachusetts, USA). The image was digitised and edge detection software (Carotid Analyzer; Medical Imaging Applications Inc, Iowa, USA) was used to trace the lumen-intima and intima-medial boundaries of the artery over a $1 \mathrm{~min}$ clip to calculate right and left cIMT. Each participant's cIMT data were analysed by two separate technicians and the two cIMT values were averaged to create a right and left cIMT score. The coefficient of variation between the two technicians' measurements was $5.3 \pm 2.6$ and $6.4 \pm 4.0 \%$, respectively, for the right and left cIMT.

Arterial stiffness and central blood pressures were assessed using the SphygmoCor CPV Central Blood Pressure/Pulse Wave Velocity System (AtCor Medical; Sydney, Australia). Briefly, a tonometer was held on the radial artery to obtain readings of the pulse waveform over $10 \mathrm{~s}$. The tonometer transduced dynamic changes in arterial force and volume into a complete pressure waveform calibrated using systolic and diastolic pressure values generated from brachial cuff measurement. A generalised transfer function gain was then applied to the pulse wave derived from the radial artery to reconstruct the aortic pulse and determine the aortic SBP as well as the pulse pressure amplification between the aorta and the radial artery. Augmentation index was calculated as the difference in pressure between the systolic shoulder of the ascending pressure curve and the systolic peak, expressed as an absolute value (augmentation pressure) and relative to a heart rate of $75 \mathrm{bpm}$ (augmentation index at heart rate $75 \mathrm{bpm}$ ).

Statistical analyses were performed with SPSS V.15.0 (SPSS, Inc, Chicago, Illinois, USA). Standard diagnostics were used to determine whether the parametric assumptions (eg, variance homogeneity, normality) of the models described below were met. Independent samples 
$\mathrm{t}$ tests were used to examine differences between the running and control groups. Correlations between continuous variables were explored using Pearson coefficients. Additional models using analysis of variance (ANOVA) (to explore the effect of gender), ANOVA (to explore the effect of continuous covariates) or multiple linear regression (to investigate the relative influence of relevant factors and their interactions in a multivariate model) were used to determine the influence of various predictors on cIMT (or other outcome variables of interest).

\section{RESULTS}

Runners and controls were comprised of equal numbers of men and women of similar ages. Runners weighed less and performed more daily vigorous physical activity (table 1). Runners also demonstrated the expected differences in many cardiovascular risk factors (table 2). There was a significant correlation between dietary intake patterns in runners and their control spouses (Block Fruit Score: Pearson coefficient $=0.38$; $\mathrm{p}=0.02$; Block Meat Score: Pearson coefficient=0.37; $\mathrm{p}=0.02$ ).

Neither left nor right cIMT differed between runners and controls $(\mathrm{p}=0.31$ and 0.53 , respectively). The left (figure 1A) and right (figure 1B) cIMT were associated with age and Framingham risk score (figure 2A,B, respectively) independent of group effects or interactions (all $\mathrm{p}>0.08$ ), and age and Framingham risk score were the only significant predictors of cIMT in a multiple linear regression model. To explore whether (in runners only) years spent running influenced the effect

\begin{tabular}{|c|c|c|}
\hline & Runners & Controls \\
\hline Sample size (n) & 42 & 42 \\
\hline Women (n) & 21 & 21 \\
\hline Age (years) & $46 \pm 13$ & $46 \pm 12$ \\
\hline Height (inches) & $67 \pm 5$ & $67 \pm 5$ \\
\hline Weight (lbs) & $149 \pm 24^{*}$ & $170 \pm 42$ \\
\hline \multicolumn{3}{|l|}{ Meds (n) } \\
\hline BP lowering & 1 & 5 \\
\hline NSAIDs & 3 & 2 \\
\hline Aspirin & 1 & 1 \\
\hline Cholesterol lowering & 2 & 4 \\
\hline Oral contraceptives & 5 & 2 \\
\hline Family history of CVD (n) & 15 & 10 \\
\hline Race time (h:min) & $4: 20 \pm 0: 47$ & - \\
\hline Running mileage & $40 \pm 16$ & - \\
\hline Years run & $12 \pm 10$ & - \\
\hline Marathons completed $(n)$ & $16 \pm 30$ & - \\
\hline Average vigorous exercise/day (h) & $2.0 \pm 1.1^{\star}$ & $0.6 \pm 0.6$ \\
\hline Average moderate exercise/day (h) & $3.9 \pm 2.2$ & $3.2 \pm 2.7$ \\
\hline Block fruit (pts) & $18.7 \pm 4.2$ & $16.8 \pm 4.5$ \\
\hline Block meat (pts) & $11.5 \pm 5.4$ & $13.1 \pm 5.8$ \\
\hline
\end{tabular}

of chronic exercise on cIMT, we controlled for years running in a partial correlation analysis of age or Framingham risk score versus the left and right cIMT. However, in this analysis, the left and right cIMT were still associated with age and Framingham risk score, suggesting that years spent running did not influence the relationships between exercise, age, disease risk and cIMT.

Aortic SBP was also not different between groups $(\mathrm{p}=0.67)$. Aortic SBP was correlated to left cIMT (Pearson coefficient $=0.32 ; \mathrm{p}<0.01$ ) and right $\mathrm{cIMT}$ (Pearson coefficient $=0.36 ; \mathrm{p}<0.01$ ) and these associations were not influenced by group effect or interactions (all p $>0.31$ ). Similar to cIMT, central SBP was associated with age and Framingham risk $(r=0.41$ and 0.52 ; both $\mathrm{p}<0.01)$ independent of group effects or interactions (all $\mathrm{p}>0.12$ ). Carotid augmentation pressure was not different between groups $(\mathrm{p}=0.67)$ and was related to age (figure 3A) and calculated Framingham risk score (figure 3B) independent of group effects or interactions (all $\mathrm{p}>0.42$ ). Carotid augmentation pressure was also not different between the two groups $(\mathrm{p}=0.07)$ when expressed relative to a heart rate of $75 \mathrm{bpm}$ (carotid augmentation index), but this parameter increased with age in both groups and was lower in runners in a multiple linear regression model (figure 4 ). There was no relationship between augmentation index and Framingham risk score (all $\mathrm{p}$ for effects and interactions $>0.20$ ).

\section{DISCUSSION}

This study was, to our knowledge, the first to assess cardiovascular risk biomarkers in trained runners versus

Table 2 Cardiovascular risk factors

\begin{tabular}{lcc}
\hline & Runners & Controls \\
\hline Left cIMT (mm) & $0.60 \pm 0.09$ & $0.62 \pm 0.11$ \\
Right clMT (mm) & $0.60 \pm 0.11$ & $0.59 \pm 0.10$ \\
SBP (mm Hg) & $130 \pm 18$ & $127 \pm 17$ \\
DBP (mm Hg) & $76 \pm 9$ & $75 \pm 10$ \\
HR (bpm) & $57 \pm 11^{*}$ & $69 \pm 12$ \\
BMI $\left(\mathrm{kg} / \mathrm{m}^{2}\right)$ & $24 \pm 4^{*}$ & $27 \pm 5$ \\
Framingham Risk (pts) & $3 \pm 4$ & $3 \pm 3$ \\
hsCRP & $0.6 \pm 0.5^{*}$ & $1.6 \pm 1.9$ \\
Total-C (mg/dL) & $181 \pm 29$ & $188 \pm 32$ \\
Non-HDL-C (mg/dL) & $114 \pm 31^{*}$ & $131 \pm 32$ \\
HDL-C (mg/dL) & $68 \pm 18^{*}$ & $58 \pm 16$ \\
LDL-C (mg/dL) & $99 \pm 27$ & $110 \pm 28$ \\
Triglycerides (mg/dL) & $76 \pm 29^{*}$ & $103 \pm 58$ \\
Central SBP (mm Hg) & $130 \pm 18$ & $127 \pm 17$ \\
Carotid AP (mm Hg) & $11 \pm 8$ & $10 \pm 6$ \\
Al@HR75 (\%) & $14 \pm 11$ & $20 \pm 11$ \\
\hline
\end{tabular}

${ }^{*}$ Significant difference between runners and controls $(p<0.05)$. AP, augmentation pressure; AI@HR75, augmentation index at heart rate $75 \mathrm{bpm}$; $\mathrm{BMI}$, body mass index; C, cholesterol; cIMT, carotid intima-medial thickness; DBP, diastolic blood pressure; HDL, high density lipoprotein; HR, heart rate; hsCRP, high sensitivity $C$ reactive protein; LDL, low density lipoprotein; SBP, systolic blood pressure. 

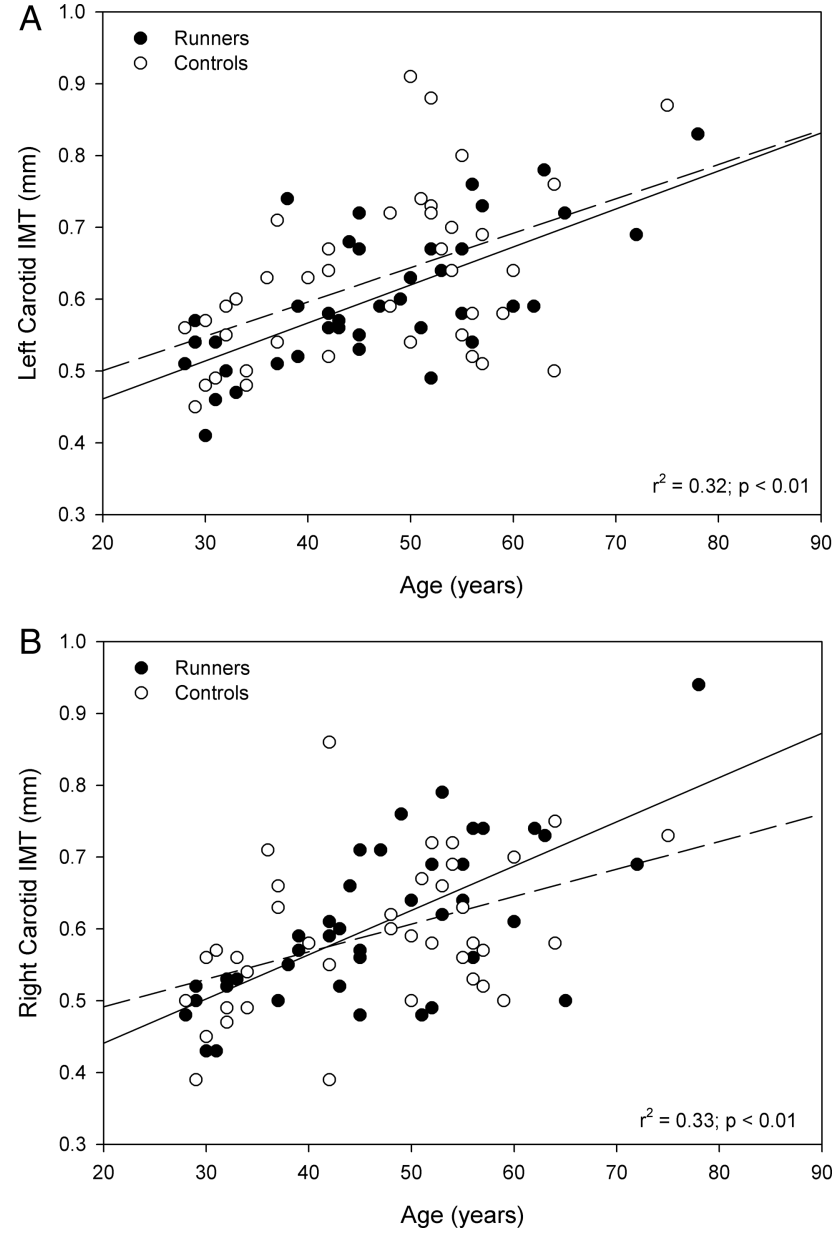

Figure 1 Relationships between age and left carotid intima-medial thickness (cIMT, A) and right cIMT (B) with data points represented for each individual participant and $r^{2}$ value shown for the entire sample. Solid line indicates regression line for runners; dashed line indicates regression line for controls.

their domestic partners to minimise the influence of lifestyle differences on the effects of chronic, high-intensity exercise. Many aspects of the cardiovascular profile were better in runners versus controls, and age and Framingham risk scores were directly related to cIMT, but cIMT did not differ between runners and controls. These results suggest that chronic endurance training improves cardiovascular risk parameters, but does not retard the progression of carotid atherosclerosis.

Habitual aerobic exercise improves many cardiovascular risk markers including body weight, ${ }^{18}$ blood lipids ${ }^{19}$ and blood pressure, ${ }^{20}$ although the individual effect is highly variable. Runners in the current study exhibited $11 \%$ lower body mass index, $63 \%$ lower CRP, $13 \%$ lower non-HDL-C, 26\% lower triglycerides and $17 \%$ higher HDL-C than controls. By contrast, neither the left cIMT nor the right cIMT differed between runners and controls. There was a similar lack of effect of marathon training on central SBP, which contributes to increases in cIMT with age. ${ }^{13}$ These data support recent
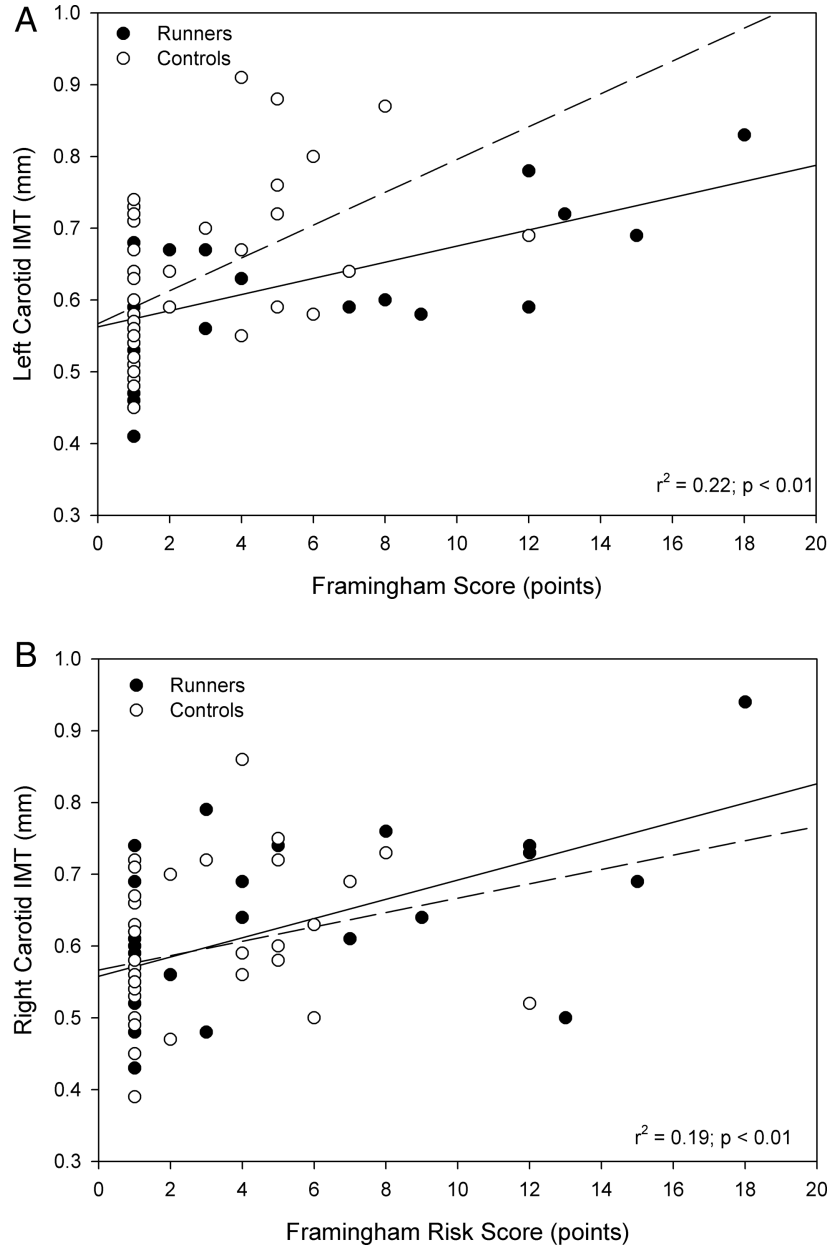

Figure 2 Relationships between calculated Framingham Risk Score and left carotid intima-medial thickness (cIMT, A) and right CIMT (B) with data points represented for each individual participant and $r^{2}$ value shown for the entire sample. Solid line indicates regression line for runners; dashed line indicates regression line for controls.

suggestions that habitual high-level physical training may reduce cardiovascular risk factors, but neither reduces nor accelerates atherosclerosis via other mechanisms such as creating vascular turbulence or influencing central blood pressure.

Age and Framingham risk score were associated with left and right cIMT and central SBP, consistent with findings from large-scale epidemiological studies. ${ }^{21}{ }^{22}$ In the current study, these relationships did not differ between trained and untrained adults, suggesting that chronic, high-intensity endurance training does not mitigate the progression of carotid atherosclerosis and intima-medial thickening associated with age and cardiovascular risk. This lack of effect was also not explained by differences in years spent running within the runner group, since controlling for duration of running history did not alter the relationships between age, disease risk and cIMT in runners. Similar findings have been reported in endurance-trained athletes with prehypertension, ${ }^{8}$ and in older female ${ }^{23}$ and male endurance athletes. ${ }^{13}$ By 

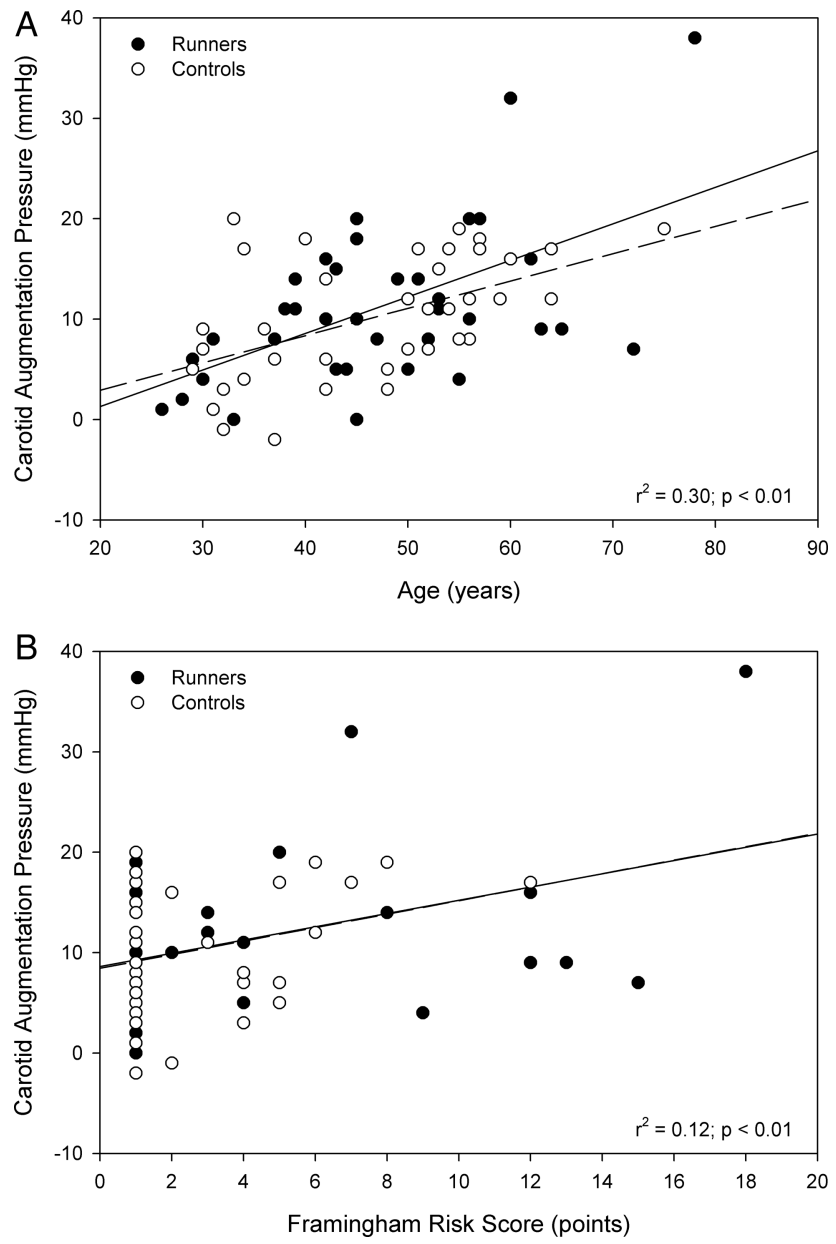

Figure 3 Relationships between age and carotid augmentation pressure $(A)$ and calculated Framingham Risk score and carotid augmentation pressure $(B)$ with data points represented for each individual participant and $r^{2}$ value shown for the entire sample. Solid line indicates regression line for runners; dashed line indicates regression line for controls.

contrast, others have documented lower cIMT values in older endurance-trained athletes, ${ }^{4} 24$ and shown that vigorous activity reduces the profession of cIMT over 3 years, ${ }^{25}$ and 6 months of endurance training lowers cIMT in healthy young men. ${ }^{26}$ Discrepancies between these various studies may be attributable to methodological differences such as participants' age and types and duration of habitual endurance training as well as the influence of confounding variables such as diet. Consequently, the current study design in which participants of a wide age range were studied in comparison with their domestic partners may better isolate the effect of chronic high-intensity endurance training on carotid atherosclerosis and cIMT.

By contrast, while augmentation pressure did not differ between groups and demonstrated the expected relationship with age and Framingham risk, controlling for heart rate (ie, assessing augmentation pressure at a uniform heart rate of $75 \mathrm{bpm}$ ) demonstrated that this calculated augmentation index was marginally lower

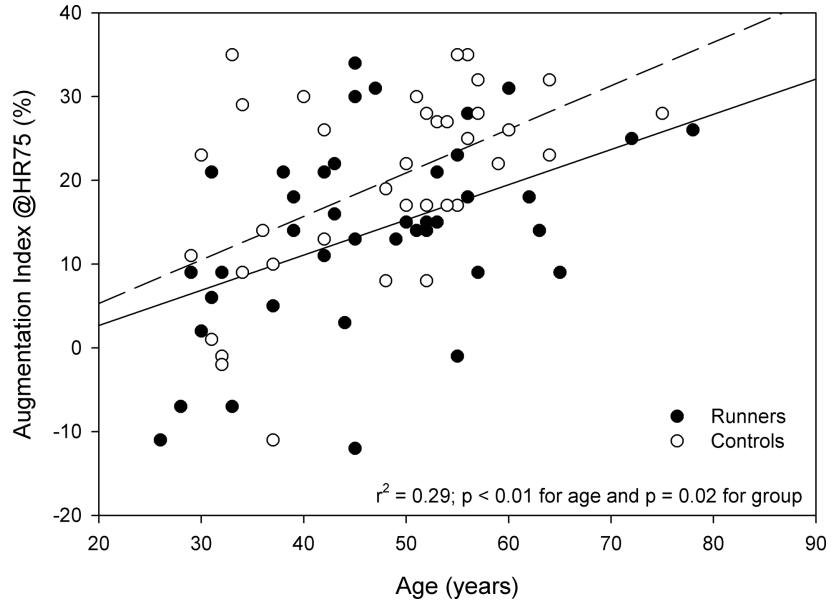

Figure 4 Relationship between age and carotid augmentation index with data points represented for each individual participant and $r^{2}$ value shown for the entire sample. Solid line indicates regression line for runners; dashed line indicates regression line for controls.

$(\mathrm{p}=0.07)$ in paired comparisons and statistically lower in a multivariate model when age was taken into account (figure 4). Augmentation pressure represents the influence of arterial stiffening on the contribution of arterial wave reflections to increasing central blood pressure. Therefore, these data demonstrate once again that chronic aerobic exercise training exerts heterogeneous effects on the vasculature, some of which may be beneficial but not sufficient to alter the progression of atherosclerotic disease.

There have been recent troubling reports suggesting that habitual, prolonged exercise and physical activity and, specifically, marathon running may actually accelerate atherosclerotic progression. For example, Kroger et $a l^{10}$ reported an unexpectedly high plaque burden in the carotid and peripheral arteries of 100 male marathoners. Similarly, coronary artery calcification scores were higher in marathoners than in non-running controls matched for Framingham Risk Score. ${ }^{9}$ The current data are reassuring since we did not find more atherosclerosis measured by cIMT in runners relative to their controls, and runners with the highest cIMTs also had the highest Framingham risk scores (figure 2). These results suggest that habitual exercise may not mitigate atherosclerotic progression, but does not exacerbate it beyond that attributable to age and risk factors.

\section{Limitations}

We assessed atherosclerosis in our participants using cIMT, but other procedures such as coronary artery calcium score might provide a better assessment of coronary and cardiovascular disease risk. ${ }^{27}$ These studies were performed in a room adjacent to the runners' exposition so that more sophisticated techniques were not available to us. Our control participants were also not entirely sedentary. Controls performed less vigorous 
exercise, but they did perform similar amounts of moderate exercise as the runners. However, this design may enhance the validity of our study because it might better isolate the influence of habitual, high-intensity exercise training on cardiovascular risk and carotid atherosclerosis.

\section{Conclusions}

Reports on the impact of long-term aerobic training on atherosclerotic risk are conflicting, and may be confounded by differences in lifestyle factors between participants. Using a comparison of runners and their non-runner control spouses, we conclude that habitual, high-intensity run training improves many aspects of the cardiovascular profile but does not reduce atherosclerosis measured by cIMT. These data are reassuring, given recent reports that marathon running may intensify atherosclerotic disease progression in central and peripheral arteries, and suggest that exercise may reduce cardiovascular events by mechanisms independent of the atherosclerotic process.

Acknowledgements The authors also gratefully acknowledge the research assistance provided by Lindsay and Judd Lorson, and William Roman and the logistical support provided by Dave McGillivray and the Boston Athletic Association; and Quest Diagnostics.

Contributors BAP, ALZ, JAC and PDT planned the study and wrote the funding proposal. BAP, ALZ, JAC, CT, ALB, PDT, PDT and KDB conducted study coordination, data collection and interpretation. BAP, ALZ, JAC, MRD and PDT wrote the article. All authors evaluated and revised the article. BAP submitted the article and is responsible for the overall content as guarantor.

Funding This research was funded by a Hartford Hospital Small Research Grant.

Competing interests PDT is a consultant for Astra Zenica International, Merck \& Company, Inc, the Schering-Plough Corporation, Takeda Pharmaceutical Company Limited, Roche, and Genomas and is a member of the speaker's bureau for Merck \& Company, Inc, Pfizer, Inc, Abbott Labs, Astra Zenica International, and the Schering-Plough Corporation.

\section{Patient consent Obtained.}

Ethics approval The study was approved by Hartford Hospital Institutional Review Board.

Provenance and peer review Not commissioned; externally peer reviewed.

Data sharing statement No additional data are available.

Open Access This is an Open Access article distributed in accordance with the Creative Commons Attribution Non Commercial (CC BY-NC 3.0) license, which permits others to distribute, remix, adapt, build upon this work noncommercially, and license their derivative works on different terms, provided the original work is properly cited and the use is non-commercial. See: http:// creativecommons.org/licenses/by-nc/3.0/

\section{REFERENCES}

1. Lorenz MW, von KS, Steinmetz $\mathrm{H}$, et al. Carotid intima-media thickening indicates a higher vascular risk across a wide age range: prospective data from the Carotid Atherosclerosis Progression Study (CAPS). Stroke 2006;37:87-92.

2. White HD, Thygesen K, Alpert JS, et al. Clinical implications of the Third Universal Definition of Myocardial Infarction. JACC 2012;60:1581-98.

3. De BG, Ambrosioni E, Borch-Johnsen K, et al. European guidelines on cardiovascular disease prevention in clinical practice. Third Joint Task Force of European and other societies on cardiovascular disease prevention in clinical practice (constituted by representatives of eight societies and by invited experts). Arch Mal Coeur Vaiss 2004;97:1019-30.
4. Galetta F, Franzoni F, Femia FR, et al. Left ventricular diastolic function and carotid artery wall in elderly athletes and sedentary controls. Biomed Pharmacother 2004;58:437-42.

5. Gando $\mathrm{Y}$, Yamamoto $\mathrm{K}$, Kawano $\mathrm{H}$, et al. Attenuated age-related carotid arterial remodeling in adults with a high level of cardiorespiratory fitness. J Atheroscler Thromb 2011;18:248-54.

6. Kim SH, Lee SJ, Kang ES, et al. Effects of lifestyle modification on metabolic parameters and carotid intima-media thickness in patients with type 2 diabetes mellitus. Metabolism 2006;55:1053-9.

7. Jae SY, Heffernan K, Fernhall B, et al. Cardiorespiratory fitness and carotid artery intima media thickness in men with type 2 diabetes. $J$ Phys Act Health 2012;9:549-53.

8. Heffernan KS, Jae SY, Tomayko E, et al. Influence of arterial wave reflection on carotid blood pressure and intima-media thickness in older endurance trained men and women with pre-hypertension. Clin Physiol Funct Imaging 2009;29:193-200.

9. Mohlenkamp S, Lehmann N, Breuckmann F, et al. Running: the risk of coronary events: prevalence and prognostic relevance of coronary atherosclerosis in marathon runners. Eur Heart $J$ 2008;29:1903-10.

10. Kroger K, Lehmann N, Rappaport L, et al. Carotid and periphera atherosclerosis in male marathon runners. Med Sci Sports Exerc 2011;43:1142-7.

11. Heffernan KS. How healthy were the arteries of Phidippides? Clin Cardiol 2012;35:65-8.

12. Kadoglou NP, Iliadis F, Liapis CD. Exercise and carotid atherosclerosis. Eur J Vasc Endovasc Surg 2008;35:264-72.

13. Tanaka H, Seals DR, Monahan KD, et al. Regular aerobic exercise and the age-related increase in carotid artery intima-media thickness in healthy men. J Appl Physiol 2002;92:1458-64.

14. Tanaka H, Dinenno FA, Monahan KD, et al. Carotid artery wall hypertrophy with age is related to local systolic blood pressure in healthy men. Arterioscler Thromb Vasc Biol 2001;21:82-7.

15. Paffenbarger RS Jr, Wing AL, Hyde RT, et al. Physical activity and incidence of hypertension in college alumni. Am J Epidemiol 1983;117:245-57.

16. Block $\mathrm{G}$, Gillespie $\mathrm{C}$, Rosenbaum $\mathrm{EH}$, et al. A rapid food screener to assess fat and fruit and vegetable intake. Am J Prev Med 2000;18:284-8.

17. Friedewald WT, Levy RI, Fredrickson DS. Estimation of the concentration of low-density lipoprotein cholesterol in plasma, without use of the preparative ultracentrifuge. Clin Chem 1972;18:499-502.

18. Donnelly JE, Blair SN, Jakicic JM, et al. American College of Sports Medicine Position Stand. Appropriate physical activity intervention strategies for weight loss and prevention of weight regain for adults. Med Sci Sports Exerc 2009;41:459-71.

19. Kelley GA, Kelley KS. Aerobic exercise and lipids and lipoproteins in men: a meta-analysis of randomized controlled trials. J Mens Health Gend 2006;3:61-70.

20. Brook RD, Appel LJ, Rubenfire M, et al. Beyond medications and diet: alternative approaches to lowering blood pressure: a scientific statement from the American Heart Association. Hypertension 2013;61:1360-83.

21. Bauer M, Delaney JA, Mohlenkamp S, et al. Comparison of factors associated with carotid intima-media thickness in the Multi-ethnic Study of Atherosclerosis (MESA) and the Heinz Nixdorf Recall Study (HNR). J Am Soc Echocardiogr 2013;26:667-73.

22. Kieltyka L, Urbina EM, Tang R, et al. Framingham risk score is related to carotid artery intima-media thickness in both white and black young adults: the Bogalusa Heart Study. Atherosclerosis 2003;170:125-30.

23. Moreau KL, Donato AJ, Seals DR, et al. Arterial intima-media thickness: site-specific associations with HRT and habitual exercise. Am J Physiol Heart Circ Physiol 2002;283:H1409-17.

24. Galetta F, Franzoni F, Tocchini L, et al. Effect of physical activity on heart rate variability and carotid intima-media thickness in older people. Intern Emerg Med 2013;8:S27-9.

25. Kozàkovà $\mathrm{M}$, Palombo $\mathrm{C}$, Morizzo $\mathrm{C}$, et al.; RISC Investigators. Effect of sedentary behaviour and vigorous physical activity on segment-specific carotid wall thickness and its progression in a healthy population. Eur Heart $J$ 2010;31:1511-19.

26. Spence $\mathrm{AL}$, Carter $\mathrm{HH}$, Naylor $\mathrm{LH}$, et al. A prospective randomized longitudinal study involving 6 months of endurance or resistance exercise. Conduit artery adaptation in humans. J Physiol 2013;591: 1265-75.

27. Yeboah J, McClelland RL, Polonsky TS, et al. Comparison of novel risk markers for improvement in cardiovascular risk assessment in intermediate-risk individuals. JAMA 2012;308:788-95.

28. den Ruijter HM, Peters SA, Anderson TJ, et al. Common carotid intima-media thickness measurements in cardiovascular risk prediction: a meta-analysis. JAMA 2012;308:796-803. 